DOI: 10.18468/rbli.2020v3n1.p10-17

\title{
Palavras em Waimiri Atroari que se Comportam como Adjetivos - Uma Discussão Preliminar
}

\author{
Words in Waimiri Atroari that behave as adjectives - a preliminary discussion
}

Resumo. Este trabalho propõe-se descrever e analisar um grupo de palavras que se comporta como adjetivos. É importante mencionar que muitas das línguas Carib não têm adjetivos como uma classe de palavras independente (Nasr 2013). Palavras que, semanticamente, seriam adjetivos são classificadas como substantivos/advérbios. O Waimiri Atroari é uma língua falada por cerca de 2.013 indígenas que habitam o norte do estado do Amazonas e sul de Roraima, Brasil. A língua Waimiri Atroari tem uma complexa e rica morfologia típica da família Carib (Gildea 1998; Meira e Gildea 2009; Santos 2007). Em bases morfológicas e sintáticas, podemos observar nesta língua cinco classes lexicais, a saber: (1) nomes incluindo pronomes, (2) verbos, (3) advérbios, (4) posposições, e (5) partículas. Além de um grupo de palavras que se comporta como adjetivos.

Palavras-Chave: Waimiri Atroari; Classe de Palavras; Adjetivos.

Abstract. This paper aims to describe and analyze a group of words that behave as adjectives. It is important to mention that many of the Carib languages do not have adjectives as an independent word class (Nasr 2013). Words that would semantically be adjectives are classified as nouns / adverbs. Waimiri Atroari is a language spoken by about 2,013 indigenous people living in the northern state of Amazonas and southern Roraima, Brazil. The Waimiri Atroari language has a complex and rich morphology typical of the Carib family (Gildea 1998; Meira and Gildea 2009; Santos 2007). On morphological and syntactic grounds, we can observe in this language five lexical classes: (1) nouns including pronouns, (2) verbs, (3) adverbs, (4) postpositions, and (5) particles. In addition to a group of words that behaves like adjectives.

Keywords: Waimiri Atroari; Word class; Adjectives

\section{Introdução}

Os Waimiri-Atroari, povo pertencente à família linguística Carib, vivem numa região dos afluentes da margem esquerda do Baixo rio Negro e do rio Amazonas, entre os estados do Amazonas e Roraima compreendendo as bacias dos rios Camanaú/Curiaú, Alalaú/Jauaperi, e uma parte da BR-174 (ManausBoa Vista). Com uma população de aproximadamente 2.013 Kinja (autodenominação dessa sociedade), distribuídas em 41 aldeias, os Waimiri Atroari realizam diversas atividades no seu cotidiano: pescarias, caça, coleta de frutos silvestres e preparação e manutenção de roçados.

Em seus roçados, eles plantam diversas espécies de mandioca, consorciada com outros tipos de tubérculos (batata-doce, macaxeira, cará) e várias espécies frutíferas. Nas suas alimentações, observam-se também uma ingestão de grandes quantidades de proteínas animais - caças diversas (anta, macacos guariba e coatá, pacas, porcos do mato, mutum, jacamim, entre outros) e peixes. (BAINES, 1991; BRUNO, 2003; 2006; 2009; 2010; 2014; DO VALE, 2002; MATAREZIO FILHO, 2010 ).

OWaimiri Atroari é, basicamente, a língua usada para a comunicação e expressão em todas as atividades 
do grupo. Os adultos só conversam entre si nessa língua, com ocasionais inserções de palavras ou expressões em português. As crianças, usualmente, chegam à escola falando apenas algumas palavras em português, mas com uma curiosidade enorme de aprendê-la. A exposição ao rádio, as visitas à Manaus para tratamento de saúde, a presença dos funcionários do Programa Waimiri Atroari e agora a possibilidade de acessar internet na área indígena sem dúvida tem contribuído para a difusão do português falado e escrito, sobretudo entre os jovens. No entanto, a língua Waimiri Atroari ainda é muito utilizada no dia-a-dia das aldeias, nos rituais e nas suas cerimônias.

Este artigo propõe-se descrever e analisar um grupo de palavras que se comporta como adjetivos. É importante mencionar que muitas das línguas Carib não têm adjetivo como uma classe de palavras independente (NASR, 2013). Palavras que, semanticamente, seriam adjetivos são classificadas como substantivos/advérbios.

\section{Morfologia nominal em Waimiri Atroari}

A língua Waimiri Atroari tem uma complexa e rica morfologia típica da família Carib (GILDEA, 1998; MEIRA E GILDEA, 2009; SANTOS, 2007). Ela apresenta tanto prefixos como sufixos. No caso, os prefixos nominais marcam posse e os sufixos uma variedade de derivações que mudam o significado dos nomes. Em bases morfológicas e sintáticas, podemos observar nesta língua cinco classes lexicais, a saber: (1) nomes incluindo pronomes, (2) verbos, (3) advérbios, (4) posposições, e (5) partículas. Além de um grupo de palavras que se comporta como adjetivos.

É importante mencionar que muitas das línguas Carib não têm adjetivo como uma classe de palavras independente (NASR, 2013; TAVARES, 2005; FERREIRA-ALVES, 2017). Palavrasque, semanticamente, seriam classificadas como adjetivos aparecem no meio do caminho entre substantivos/verbos/ advérbios (GIVÓN, 2001). Estudos tipológicos (SHOPEN, 1992; CROFT, 1990; DIXON, 1982) discutem que adjetivo não é uma classe universal de palavras. De acordo com Meira e Gildea (2009, p. 131), "antes de se posicionar a respeito de uma classe específica de adjetivos e/ou advérbios nas línguas da família Carib, é importante verificar com cuidado as propriedades morfológicas e sintáticas que cada uma dessas línguas atribui às classes de nome e verbo."

Tendo como base as observações desses autores, apresento uma discussão preliminar sobre alguns aspectos morfológicos e sintáticos para pensar o comportamento deste grupo de palavras em Waimiri Atroari. Palavras que designam propriedades, qualidades ou estados que são relativamente estáveis ao longo do tempo. Elas geralmente precedem o substantivo, mas também ocorrem após o nome. $E$, são morfemas livres formados por apenas uma raiz.

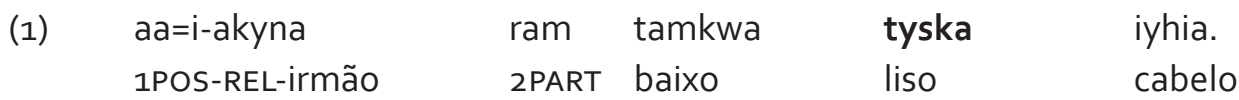

'Meu irmão é baixo e tem cabelos lisos.'

(2) tapyryma iyhia.

preto cabelo

'O cabelo é preto.' 
(3)
Maiwu
wykyry mepiri
wu-pia
gordo
homemanta
matar-P.IMD

'O homem gordo matou a anta'

(4) aa=papa ram sehsa tydapra

1POS-pai 2PART alto gordo (gorduroso)

'Meu pai é alto e gordo.'

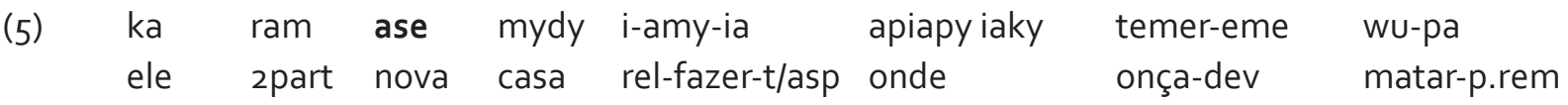
'Ele fez a aldeia nova onde ele matou a onça.'
n-aryme-pa sakene-pa wahpary
iakapa n-aryma-pa ty-mydy
taka
3s-voltar-p.rem bravo-enf muito
depois 3 s-voltar-p.rem 3 reflx-casa
al
'Voltaram muito bravos para a aldeia deles.'

Na lista abaixo, apresentamos um pequeno corpus do grupo de palavras que se comporta como adjetivos e depois exemplificamos algumas delas em contextos sentenciais:

\begin{tabular}{ll|ll} 
Waimiri Atroari & Português & Waimiri Atroari & Português \\
$\begin{array}{l}\text { Abemyhpa } \\
\text { Aberymyhy abemyhy }\end{array}$ & redondo & Sakyna & raivoso \\
Anykyxi & fino & Saweny sewuna & leve \\
Ase & novo & Tehe & alto \\
Awinihe awinini, & um, sozinho & curto para pessoa \\
awinjehe, awenjahky & & Taha & grande, líder \\
Ba'xiri be'xiri & pequeno & Tamxa & macio, mole, preguiçoso \\
Bahinja baxinja & pequeno, pouco, criança & Tapany typany tipyna & duro \\
Bakyma & azedo, salgado & Tapyryma & preto, escuro \\
Byryryma & torcido,torto & Temyna & seco \\
Djapyma & direito, certo, correto & Texiba & triste \\
Etypa & quente & Teximy & ruim, gosto ruim \\
Inama & fraco (a) & forte, potente \\
Kareme, karany & bom, bonito (a) & Trika & ágil, rápido \\
Kyby & curto para animais & Tuwaka & feliz, contente \\
Kybyma & e plantas & & \\
Kykyryhpa & amargo & Tuwera & maduro \\
Mady madym & queimado & Txamyry & velho, ancião \\
Maiwu maiy & aleijado & Txanpa & grávida \\
Masara & gordo (a), grosso (a) & Txika & afiado \\
Mixopy & preguiçoso, aprendiz & Txipikia & envergonhado \\
& longo, comprido & Txitymy & solteiro, sozinho
\end{tabular}




\begin{tabular}{ll|ll} 
Nerimy & bravo, corajoso & Tybyska & liso, macio \\
Nyryny & vivo & Tykomia & frio, gelado \\
Panapy & trabalhador & Tykoxinja & sujo \\
Panaxi & danado & Typyra & fedorento, mau cheiro \\
Pine & Curto & Waina & fino para pessoa \\
Pitymy & Solteiro & Woxypia & pesado \\
Sakra & branco & Xiwia & bom, bonito, amarelo \\
& & & e vermelho
\end{tabular}

Ao contrário dos substantivos, as palavras que se comportam como adjetivos nesta língua não podem receber o sufixo absentivo $\{-m y\} q u e$ indica 'ausência'.

$\begin{array}{ll}\text { (7) ety-my 'sem nome' } & \text { emy-my 'sem pênis' } \\ \text { iee-my 'sem dente' } & \text { iaska-my 'sem parentes' } \\ \text { iyhia-my 'sem cabelo, careca' } & \text { eba-my 'sem olho/cego' }\end{array}$

Além disso, apenas elas podem receber o sufixo enfático \{-pa\} (por exemplo, tamkwa 'curto/baixo'; tamkwa-pa 'muito curto/baixo'; kwada 'feio, ruim' kwada-pa 'muito feio, muito ruim'). Algumas destas palavras que se comportam como adjetivos, também podem se comportar como nomes. Ver casos abaixo:

(8) bahinja $\sim^{1}$ baxinja pequeno, pouco, criança

(a) Tuwadja wehma ram bahinj-eme Damixiri wehp-eme kapy-ma.
Tuwadja flecha 2part pequena-dev Damixiriflecha-dev neg-?

'A flecha de Tuwadja é menor que a flecha de Damixiri.'

$\begin{array}{lllll}\text { (b) Dauna } & \text { bahinja } & \text { xiba } & \text { myryky-piany } & \text { araky. } \\ \text { Dauna } & \text { pouco } & \text { peixe } & \text { pescar-p.rec } & \text { hoje }\end{array}$

'Dauna pescou poucos peixes hoje.'

(c) bahinja n-itxi-pia mydy i-akyna-se.

Criança 3s-ir-p.imd casa rel-varrer-no propósito de

'As crianças foram varrer a casa.'

(d) bahinja ram ariwi i-wak-e.

Criança 2part surubim rel-segurar-t/asp

'A criança está segurando o surubim.'

(e) naminja ny-tyte-pa bahinja pyna.

cachorro 3s-chegar-p.rem criança perto/próximo

'O cachorro chegou perto da criança'

1 Este símbolo " " é indicação de variação livre; podemos encontrar as duas formas. Não está necessariamente relacionado à diferença dialetal. 
(f)

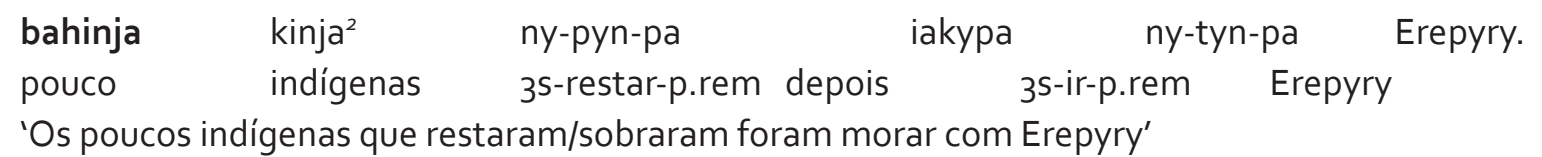

(9) taha

(a) Paryry kama taha Xeri Kaapa wata'.

grande, líder

Paryry $r$ oça 2part grande Xeri roça como

'A roça do Paryry é tão grande quanto a roça do Xeri.'

(b) pana a'a n-itxi-piany taha kanuwa ta

Ontem 1+3pro 1+3s-ir-p.rec grande canoa loc

warara bi pipe-se.

tartaruga ovo procurar-no propósito de

'Ontem, fomos na canoa grande procurar ovos de tartaruga.'

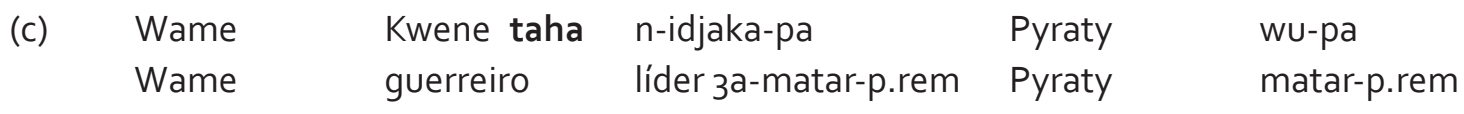

Piawuna many Kiawuna many.

Piawuna também Kiawuna também

'Wame, o líder guerreiro, matou Pyraty, Piawuna e Kiwauna também.'

(d) taha iakaha ehry-ky!

grande desenho pintar-imp

'Pinte o desenho maior!'

(e) Aa ram minja pyna hy-kyt-ia maia taha ke.

1pro 2part mandioca 1-cortar-t/asp faca grande instr

'Eu vou cortar mandioca com a faca grande.'

(f) Waika samka ram taha.

Waika rede 2part grande

'A rede de Waika é grande'

(g) kinja iky taha Dakwa.

indígena iky líder Dakwa.

'Dakwa era o líder dos índios Iky'

Sintaticamente, este grupo de palavras, assim como os nomes, podem ocupar a posição de sujeito ou objeto. Por outro lado, não está claro se é possível, de fato, usar a partícula de segunda posição ram como um constituinte limite em sintagmas do tipo <ADJ N>. É necessário fazer mais testes, tendo em vista que nem sempre os falantes Waimiri Atroari permitem o tipo de construção como se mostra nos exemplos (10) e (11) abaixo:

2 Como os Waimiri Atroari se auto-denominam. Mas também é utilizado para indígena . 


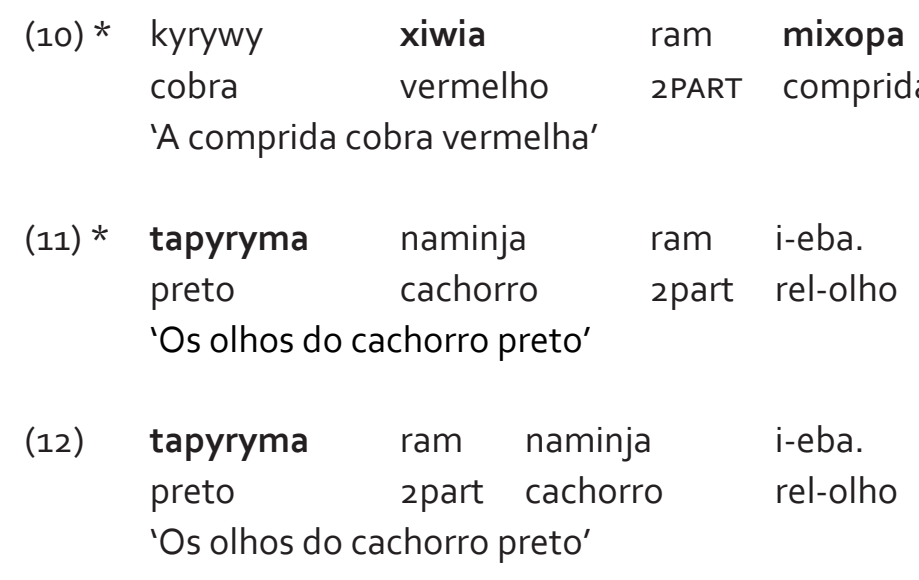

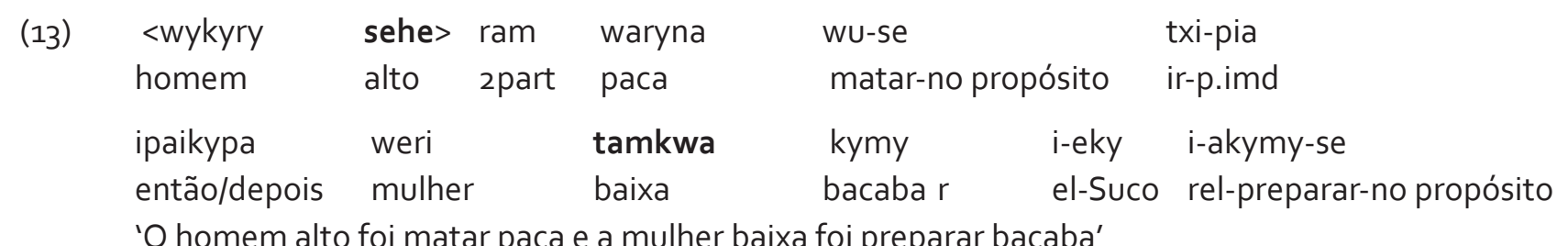

\section{Considerações Finais}

No presente trabalho apresentamos uma descrição e discussão sucinta e preliminar sobre este grupo de palavras, como elas se comportam morfologicamente e sintaticamente. Como já foi observado para outras línguas Carib, nesta língua os nomes e os verbos não são problemáticos e são fáceis de identificar. No entanto, as classes restantes, tais como as palavras que se comportam como adjetivos, advérbios e posposições, são muito mais difíceis de caracterizar.

\section{Abreviações}

\begin{tabular}{ll|ll} 
A & 'Sujeito de verbo transitivo' & P.REM & 'passado remoto' \\
ADJ & 'adjetivo' & PRO & 'pronome' \\
AL & 'alativo' & POS & 'possessivo' \\
DEV & 'devaluativo' & 'reflexivo' \\
ENF & 'enfático' & SEL & 'prefixo relacional' \\
IMP & 'imperativo' & T/ASP & 'sujeito de verbo transitivo' \\
INSTR & 'instrumental' & 2 PART & 'tempo/aspecto' \\
LOC & 'locativo' & 1 & 'partícula de segunda posição \\
N & 'Nome' & 2 & 'primeira pessoa singular \\
NEG & 'Negação' & 3 & 'segunda pessoa sing. or pl.' \\
P.IMD & 'passado imediato' & 'terceira pessoa sing.or pl.' \\
P.REC & 'passado recente' & $1+3$ & 'primeira pessoa plural exclusiva'
\end{tabular}




\section{Referências Bibliográficas}

BAINES, Stephen G. "É a funai que sabe": A frente de atração Waimiri-Atroari, Belém: MPEG/CNPQ/ SCT/PR, 1991.

BRUNO, Ana Carla. Waimiri Atroari grammar: Some phonological, morphological, and syntactic aspects (Ph.D. dissertation). University of Arizona. 2003.

BRUNO, Ana Carla. The causative construction in Waimiri Atroari. Liames - Línguas Indígenas Americanas v. 6, p. 101-108, 2006. Disponível em <https://periodicos.sbu.unicamp.br/ojs/index.php/ liames/article/view/1448/0>.

BRUNO, Ana Carla. Phrase structure, clauses, and word order in Waimiri Atroari (Carib Family). Revista Virtual de Estudos da Linguagem-REVEL, v. 7, n. 3, pp. 1-13, 2009a. Disponível em <http://www.revel. inf.br/files/artigos/revel special 3 phrase structure.pdf>

BRUNO, Ana Carla. Educação indígena e questões linguísticas: Quando a ortografia torna-se um problema - a experiência Waimiri Atroari. Moara, v. 32, pp. 109-120. 200gb. Disponível em <http:/l periodicos.ufpa.br/index.php/moara/issue/view/233/showToc>

BRUNO, Ana Carla. How can I write my language? Linguistic analysis and language revitalization: lessons from Waimiri Atroari syllable structure. liames - Línguas Indígenas Americanas, v. 10, pp. 85-99. 2010. Disponível em <https://periodicos.sbu.unicamp.br/ojs/index.php/liames/article/ view/1510doi:10.20396/liames.v10i1.1510>

BRUNO, Ana Carla (Org.). Vozes da Floresta: a arte de contar histórias - Histórias do passado e do cotidiano indígena. Manaus: Editora Inpa, 2014.

CROFT, William. Typology and universals. Cambridge: Cambridge University Press, 1990

DIXON, R. M. W. Where have all the adjectives gone? The Hague: Mouton de Gruyter, 1982.

DO VALE, Maria Carmen Rezende. Waimiri-Atroari em festa é maryba na floresta. 2002. Dissertação (Mestrado em Sociedade e Cultura na Amazônia), Universidade Federal do Amazonas, Manaus, 2002.

FERREIRA-ALVES, Ana Carolina. Morfofonologia, morfossintaxe e o sistema de tempo, aspecto e modo em Arara (Karib). 2017. Tese (Doutorado em Semiótica e Linguística Geral), Universidade de São Paulo, São Paulo, 2017.

GILDEA, Spike. On reconstructing grammar: Comparative Cariban morphosyntax (Oxford Studies in Anthropological Linguistics18). Oxford: Oxford Press, 1998.

GIVÓN, T. Syntax: An Introduction. 2 vols. Amsterdam: John Benjamins, 2001

MATAREZIO FILHO, Edson Tosta. Ritual e pessoa entre os Waimiri Atroari. 2010. Dissertação (Mestrado em Atropologia Social), Universidade de São Paulo, SãoPaulo, 2010. 
MEIRA, Sérgio. A grammar of Tiriyó. 1999. Thesis (Ph.D.). Rice University, Houston, 1999.

MEIRA, Sérgio. Word class systems in Cariban languages. Manuscrito, 2002.

MEIRA, Sergio; GILDEA, Spike. Property concepts in the Cariban family: Adjectives, adverbs and/or nouns? In WETZELS, L. W. (Ed.). The linguistics of endangered languages: Contributions to morphology and morphosyntax, pp. 95-133. Utrecht: lot. 2009. Disponível em <https://dspace.library.uu.nl/ bitstream/handle/1874/297134/bookpart.pdf? sequence $=2>$

NASR Kalek, Fairuz. La frase nominal em Ye'kwana. UniverSOS, v. 10, pp. 53-71. 2013. Disponível em < https://www.uv.es/ calvo/amerindias/numeros/n10.pdf>

PACHECO, Frantomé Bezerra. Aspectos da gramática Ikpeng (Karíb). 1997. Dissertação (Mestrado em Linguísttica), Universidade Estadual de Campinas, Campinas/SP, 1997. Disponível em < $<$ http:/l repositorio.unicamp.br/bitstream/REPOSIP/270876/1/Pacheco Frantome Bezerra M.pdf>

SANTOS. Gesalma Mara F. Morfologia Kuikuro: Gerando nomes e verbos. 2007. Tese (Doutorado), Universidade Federal do Rio de Janeiro, Rio de Janeiro, 2007

SHOPEN, Timothy (Ed.). Language typology and syntactic descriptions. Cambridge: Cambridge University Press, 1992.

TAVARES, Petronila. A grammar of Wayâna. 2005. Thesis (Ph.D.), Rice University, Houston, . 2005 